\title{
Oxygen-ozone therapy in the treatment of tissue adipose diseases
}

\author{
Gaetano Cuccio, ${ }^{1}$ Marianno Franzini ${ }^{2}$ \\ ${ }^{1}$ Doctor II Master Oxygen-Ozone University of Pavia, Pavia; ${ }^{2}$ Oxygen-Ozone Therapy Scientific \\ Society, Gorle (BG), Italy
}

\begin{abstract}
The panniculosis or panniculopathy edematous fibro sclerotic (PEFS), commonly called cellulite is a subcutaneous adipose disease that afflicts the vast majority of women at all ages. PEFS is framed as a subcutaneous adipose suffering from venous and lymphatic stasis whose etiology is multifactorial. Many others are the implications and clinical relapses of diseases of inflammatory or autoimmune basis that determine disease states with involvement of the patient's general conditions. The oxygen-ozone therapy, thanks to its abilities of improving the rheological properties of the microcirculation, immuno-modulating and anti-inflammatory abnormalities, arises as adjuvant and is a valid method, which is also alternative compared to conventional protocols.
\end{abstract}

\section{Introduction}

With this work I want to expose and support the thesis that the oxygen-ozone $\left(\mathrm{O}_{2}-\mathrm{O}_{3}\right)$ therapy is a broad therapeutic application in diseases of the adipose tissue of local and systemic interest, tracing an exhibition on the possible applications in various pathological conditions.

Inflammatory or degenerative events affecting the subcutaneous tissue are characterized by alterations of the fat cells, stromal and vascular system. ${ }^{1}$ They can lead to hypertrophy, atrophy, disintegration and necrosis of the adipose tissue. ${ }^{2}$ Examples of lipodystrophy are the Barraquer-Simon disease, a nodular fever characterized by inflamma-

Correspondence: Gaetano Cuccio, Doctor II Master Oxygen-Ozone University of Pavia, Oxygen-Ozone Therapy Scientific Society, Via Roma 69, 24020 Gorle (BG), Italy.

Tel: +39.091.664645. E-mail: gaetanocuccio28@libero.it

Key words: PEFS; Cellulite; Oxygen-ozone; Adipose; Microcirculation.

Conflict of interest: the authors declare no potential conflict of interest.

Received for publication: 21 June 2016.

Accepted for publication: 25 July 2016.

(C) Copyright G. Cuccio and M. Franzini, 2016

Licensee PAGEPress, Italy

Ozone Therapy 2016; 6270

doi:10.4081/ozone.2016.6270

This article is distributed under the terms of the Creative Commons Attribution Noncommercial License (by-nc 4.0) which permits any noncommercial use, distribution, and reproduction in any medium, provided the original author(s) and source are credited. tory nodules under the skin with matching surface of erythema, ${ }^{3}$ the adiponecrosis that can be caused by crushing, freezing, injections of lipolytic drugs, continuous mechanical injuries such as those caused by a violent percussive massage or skin application of machinery, whose symptomatology is characterized by inflammation pain, erythema, hemorrhage, and finally tissue necrosis. ${ }^{3,4}$ This brief exposure includes panniculosis or panniculopathy edematous fibro sclerotic (PEFS) commonly called cellulite.

Today, $\mathrm{O}_{2}-\mathrm{O}_{3}$ therapy is recognized by many as an excellent and effective medical method for the treatment of several diseases. Several countries around the world practice it in their own hospitals, clinics and universities, acting on various diseases.

For a long time fat has been considered little involved in the processes that regulate the body, relegating it to purely thermal and mechanical barrier function as well as to that of storage and energy storage in the form of triglycerides, and then returning it as free fatty acids according to the body's needs. ${ }^{1}$

Today, in the light of current research and results, it is considered a real vocation in endocrine tissue, involved in many metabolic mechanisms by which it exercises fundamental roles for the production and synthesis of complex signals involved in the conversation between even distant organs. ${ }^{2}$ In obesity, the amount of impaired secretion of adipokines determines important circulatory and metabolic disorders, acting as a co-factor in pagenesi of cardiovascular disease and metabolic syndrome. ${ }^{1,2}$

\section{Oxygen-ozone therapy}

The treatment with $\mathrm{O}_{2}-\mathrm{O}_{3}$ uses a gas mixture with different concentrations of $\mathrm{O}_{3}$, establishing itself as an effective and versatile technique available to the clinicians.

Ozone is an allotropic form of oxygen in the atmosphere that absorbs most of the ultraviolet radiation emanating from the sun, allowing on the surface life.

The $\mathrm{O}_{2}-\mathrm{O}_{3}$ therapy is a macroterapia that reactivates microcirculation. ${ }^{5}$ It works by improving the oxygenation in different tissues, and exerts a protective multi-organ action.

The therapeutic effects of $\mathrm{O}_{2}-\mathrm{O}_{3}$ mixtures are due to a controlled oxidative stress, ${ }^{6}$ which triggers as in a paradox many metabolic factors, in relation to the pathological picture and the predicted clinical outcome.

The $\mathrm{O}_{2}-\mathrm{O}_{3}$ possesses powerful anti-edema and anti-inflammatory activity being widely endeavored in diseases characterized by inflammation affecting the arterial circulatory system, venous and lymphatic.,6

Ozone possesses anti-inflammatory action due to the reduction of the production of prostaglandins by acting on the synthesis of arachidonic acid and also has an antioxidant action for activation of the protective enzymatic functions of endogenous cells against free radicals. ${ }^{7,8}$

The use of $\mathrm{O}_{2}-\mathrm{O}_{3}$ in youth is benign. Hypodermatitis causes an 
immediate benefit, with an obvious reduction of edema due to increased lymphatic drainage activities and improved perfusion of the vessels. The reduced capacity in the functioning of the microcirculation causes, with the relative slowdown of the blood and lymphatic circulation, an excessive accumulation of cellular catabolites in the tissues, creating a sort of self-intoxication and greater permanence of free radicals, with consequent damage of the cell structures and tissue aging. ${ }^{8}$

The experience together with continuous research have identified for the therapeutic window ${ }^{8,9}$ optimal concentrations of $\mathrm{O}_{2}-\mathrm{O}_{3}$, processed with the efficacy and safety criteria as those reported by the protocols provided by the Società Scientifica di Ossigeno Ozono Terapia.

The therapy with $\mathrm{O}_{2}-\mathrm{O}_{3}$ is considered as an absolute medical method with less risk and fewer side effects for the patient.

\section{Mechanisms of action}

Ozone is an unstable molecule that, dissolved in aqueous solution, reacts instantly with substrates such as unsaturated fatty acids, ${ }^{5,9}$ with reducing compounds such as reduced glutathione and some water soluble proteins such as uric acid, ascorbic acid, albumin, glucose, and those cysteine-rich. 6,8

The biochemical reactions determinate the formation of an excess of $\mathrm{H}_{2} \mathrm{O}_{2}$ that spreading in cells activates many metabolic pathways. At the same time the excess of $\mathrm{H}_{2} \mathrm{O}_{2}$ is reduced by the intracellular antioxidant system..$^{8,9}$ Our thesis is inspired by the observations of the effects, which produces $\mathrm{O}_{2}-\mathrm{O}_{3}$ therapy in patients with disorders of the fat tissue with different etiology.

The $\mathrm{O}_{2}-\mathrm{O}_{3}$ therapy applications in the treatment of antiviral infections collect successes due to the ability of the ozone to modulate, in the acute phase, the synthesis of immunoglobulins, particularly IgM, through the activation of immunocompetent cells of the anti-infection defenses complementary to those specifications. ${ }^{5,7,8}$

The mechanism of action of $\mathrm{O}_{2}-\mathrm{O}_{3}$ about viruses can be attributed to oxidative capacity and the consequent inactivation of specific viral receptors used to create the bond with the wall of the cell to infect. In other words, while in bacteria the $\mathrm{O}_{3}$ acts with destructive action, in the virus it is inhibited by the adhesion mechanisms of cell reproduction before the attack. ${ }^{5}$

\section{Mechanisms of action on inflammation of oxygen-ozone}

Histopathological events dictated by immune and hemodynamic conditions suggest a broad use of therapy with $\mathrm{O}_{2}-\mathrm{O}_{3}$.

The immunomodulatory properties, anti-inflammatory, antiviral, antibacterial, and the ability to improve perfusion of the microcirculation and thus restore a proper metabolic conversation between the vessel system, the functions and the neighborhood, make this method probably elective to afford clinical conditions involving the adipose tissue as a forum for primary lesions, as predisposing factor in the pathogenesis of some metabolic diseases, or as a target in metabolic dramatic events as the steatonecrosis pancreatic or acute hemorrhagic pancreatitis. $^{9,10}$

As it is known, the $\mathrm{O}_{2}-\mathrm{O}_{3}$ is used for the intense immunomodulatory action. In fact, it was demonstrated that blood samples incubated with $\mathrm{O}_{2}-\mathrm{O}_{3}$ mixtures showed increased production of interferon-y (IFN- $\gamma$ ) and transforming growth factor $\beta 1$ (TGF- $\beta 1$ ). ${ }^{9}$ The increase in non-specific immune defenses has been characterized by the increased presence of
INF- $\gamma$ so, as it has been found, is the release of IFN- $\beta$ and other cytokines such as interleukin (IL) 2,6,8, tumor necrosis factor (TNF- $\alpha$ ), TGF- $\beta$ and a granulopoietina (GM-CSF)..$^{5,7-9}$

Another fundamental property of $\mathrm{O}_{3}$ is realized on the metabolism of carbohydrates, proteins and on fatty acids. The aerobic demolition of glucose in therapy $\mathrm{O}_{2}-\mathrm{O}_{3}$, increases the availability of adenosine triphosphate (ATP) that is most required in degenerative and inflammatory diseases, and intervenes in the metabolism of protein for its affinity for sulfhydryl groups, reacting with essential amino acids such as methionine and tryptophan or cysteine that contain sulfur., ${ }^{5,7}$ In the unsaturated fatty acid it is linked to the double carbon bond, transformed into water-soluble compounds. ${ }^{5}$

$\mathrm{O}_{3}$ favors the formation of peroxides exerting antibacterial effect with a mechanism similar to that used by white blood cells used to bacterial phagocytosis. With viruses it has a virustatic action by preventing their adherence to the cellular receptors. ${ }^{5}$ The formation of peroxides intervenes on erythrocyte metabolism. The $\mathrm{O}_{3}$ develops an interaction with the double bonds of unsaturated fatty acids of the layer of the phospholipids in the erythrocyte membrane by increasing the deformability, thereby reducing blood viscosity, increases the production of 2.3-diphosphoglycerate, responsible of the $\mathrm{O}_{2}$ supply of the hemoglobin to tissues, with better perfusion and transport along the vascular beds. .,8,11

$\mathrm{O}_{3}$ has an anti-inflammatory action by reducing the production of proinflammatory cytokines IL-2, IL-4, IFN- $\gamma$ TNF- $\alpha$, Th2 activation and suppression of Th1, the ratio CD4+/CD8+ $(\mathrm{P}<0.05)$ with $\mathrm{CD} 3+$ human leukocyte antigens-idiosyncratic drug reactions constant value in the multiple sclerosis, ${ }^{12}$ immunoglobulins and inflammatory mediators as well as circulating immune complexes, antioxidant enzymes such as catalase, the superoxide dismutase, glutathione, it also reduces C-reactive protein, total cholesterol, low-density lipoprotein and triglycerides and homocysteine and finally increases the high-density lipoprotein. ${ }^{5-8}$

The $\mathrm{O}_{2}-\mathrm{O}_{3}$ acts on the cell-mediated inflammatory response ${ }^{7}$ due to improved tissue oxygenation and cellular districts, inhibiting: activation and leukocyte and platelet adhesion, activation of phospholipase A2 that with its enzymatic action on food on arachidonic acid increases the amount of prostaglandins and leukotrienes, the cyclooxygenase and metalloprotease, by neutralizing endogenous reactive oxygen species and stimulating local production of antioxidant enzymes..$^{5,7,8}$

$\mathrm{O}_{3}$ causes immediate analgesic effect in the inoculation site for inactive and destroys algogenic substances, it inactivates bradykinin, alters serotonin which has an indole nucleus, and produces a long-lasting analgesic effect through the denaturation of some cellular proteins such as kininogen and kallikrein, alteration of receptor sites for algogenic substances and increased gene expression for the antinociceptive activity. ${ }^{7-9}$

\section{Body fat}

Adipose tissue is considered by many scholars as a true common organ for its diverse endocrine and metabolic functions. ${ }^{1,2}$ Adipose tissue is found primarily in the subcutaneous layer where it forms panniculus of various thickness, consisting of aggregations of adipocytes thickly pushed together and wrapped in a web of reticular fibers, divided into lobules by connective tissue septa, where there is a dense network of vessels with each adipocyte is in contact. ${ }^{13}$ Since a few years it has been shown that the fat is not only a warehouse responsible for the conservation of the excess calories in the form of triglycerides or the release of fatty acids according to the needs. ${ }^{1}$ There is a direct relationship between the increase in body fat and the production of fatty acids (FFA), hormones, and proaterogene of proinflammatory molecules, which together are involved on complications associated with obesity, diabetes, hypertension and dyslipidemia, coming to the metabolic syn- 
drome. ${ }^{1,2}$ The greatest amount of proinflammatory molecules would seem to be issued by the nonfat cells of the adipose tissue itself, such as eosinophilic cells, monocytes and macrophages. With the exception of leptin and adiponectin only secreted by adipocytes, the release of interleukins and a number of inflammatory cytokines, such as the production of growth factors and defense, are more chargeable to other subcutaneous cellular components. The tissue suffering caused by proinflammatory molecules determines a state of cellular hypoxia, with consequent increase of angiogenesis, the greatest amount of connective tissue, the number of fibroblasts and macrophages. ${ }^{1,2}$

In overweight, the morphological and functional alterations of the subcutaneous tissue do not affect only the adipocyte. Through neural nutritional hormonal and paracrine mechanisms the increase of adipogenesis stimulates the recruitment and proliferation of mesenchymal precursors - preadipocytes - and their subsequent differentiation into mature forms Fat Cells. ${ }^{1,2}$

The inflammatory response leads to the alteration of the tissue components, for the most part of those relevant to the extracellular matrix exacerbated by endothelial fibrosis. ${ }^{14}$

\section{Anatomical memories: the fatty tissue}

Adipose tissue is $15-20 \%$ of total body weight. The amount is widely variable in relation to age, gender, body site and nutritional status. Many features, among which that of mechanical protection and thermal insulation body, act also as a storage site and reserves of energy metabolites. The ability of adipose tissue to accumulate triglycerides is virtually unlimited, and adipocytes may undergo hypertrophy by accumulation in the order of thousands of times. The adipose tissue intervenes in the mechanisms of thermogenesis and in the maintenance of body temperature and is capable of secreting nature of hormone or hormone-like substances. ${ }^{1}$

There are two types of adipose tissue the so-called white to yellowish color due to the presence of pigmented substances such as carotenoids and bile pigments and the brown, dark in color because of a large number of mitochondria, but little expressed in the adult and with exclusively thermogenic functions.

White adipose tissue is distributed primarily in the subcutaneous tissue around the kidney, mesentery, in the yellow bone marrow, in the retroperitoneal regions, axillary and inguinal, between the fibers of skeletal muscle or around the myocardium. It is almost non-existent on the eyelids, the ear and the scrotum and reaches its maximum thickness at the gluteal region.

When observed it is of yellowish color, due to the presence of pigmented substances such as carotenoids and bile pigments. It presents a scaffold connective, coming from the dermis, in its turn connected to the underlying muscle groups, organized in different thickness baffles which delimit the lobules organized in globular clusters composed by cells of mesenchymal origin, adipocytes or fat cells.

In the subcutaneous adipose tissue, we can distinguish a superficial areolar layer and a deep lamellar layer. In the first level lobules tend to assume a round shape, in the second they are flattened with the major axis parallel to the body surface, separated from connective retinacula and also oriented according to the cutaneous plane. ${ }^{15}$ The laminated layer is a moving area, which allows the sliding of the skin at the musculoskeletal level.

The amount of subcutaneous fat differs in relation to nutritional status, age and gender. The regional distribution is a secondary sexual character, determined by secreted steroid hormones and especially from the gonads.

In the female, after puberty, there is an increase in subcutaneous fat levels, mainly in the buttocks, hips, thighs and breasts. In the male, lipid deposits are mostly distributed on the trunk and abdomen. Sex and its endocrine framework also affect the organization of the adipose tissue, resulting in histological differences. ${ }^{14}$ The dermal-hypodermic boundary is, therefore, characterized by a hill profile that, even before the development of cellulite (a typically female imperfection) can be highlighted by putting in traction the connective inextensible branches (Figure 1). ${ }^{15}$

In the case of obesity, the lobules increase in volume but are held back, along the edges, by the branches around them. Their apexes protrude into the dermis, as dome-shaped pads (fat buds) creating the hated blemish skin of so-called orange peel. ${ }^{15}$ The unsightly alternating depressions and elevations are evident at first only during standing, even in recumbency.

Conversely, in the male subcutaneous, the branches of the areolar plan assume an oblique course, crossing to delimit smaller lobules, of polygonal shape, which, even in the case of hyper-lipid accumulation, does not tend to protrude toward the dermis.

The brown adipose tissue, which is found only in mammals, is predominantly localized in the subcutaneous interscapular region, axillary, perivisceral outside the hypodermis, along the large blood vessel, around the kidney and adrenal gland, appearing in the fresh preparations with a dark coloring due to the rich vasculature and the presence of numerous cytochromes placed on mitochondria.

In women, the connective branches in the areolar layer have an arrangement which is perpendicular to the cutaneous plane, separating bulky lobules in rectangular section, whose quotes press against the reticular dermis and will protrude in the form of fat buds of hemispherical shape. ${ }^{15}$

\section{Production of adipokines in adipose tissue}

In obese, as well as in localized accumulations of fat, the adipocyte hypertrophy, therefore usually a higher energy intake, sets in motion at cascade profologistic processes causing increased vascularization of adipose tissue with macrophage infiltration, release of inflammatory cytokines, insulin-local resistance, accelerated lipolysis with the release of FFA, decreased production of adiponectin and increased production of leptin. ${ }^{1,2}$ Today it is clear that the altered secretion of adipokines in obesity, especially abdominal, as well as in localized fat, determines important circulatory and metabolic disorders. In visceral fat tissue and subcutaneous, especially in the obese, in particular inflammation and edema of stasis conditions, we are seeing increased

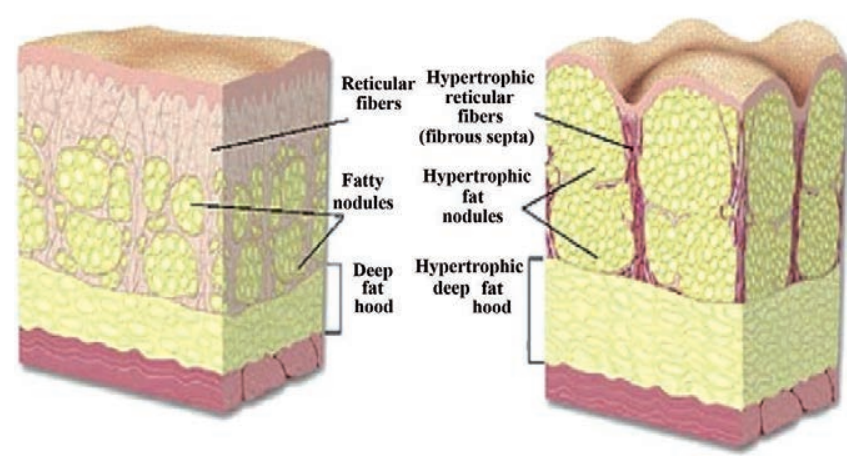

Figure 1. Organization of subcutaneous adipose tissue in the male (left) and female (right) gender. In humans, the branches that delimit the lobules of the connective areolar layer assume an oblique course, while in the woman are arranged in an orthogonal manner to the cutaneous plane characterizing the classic bill profile of the dermo-hypodermic border. 
production of interleukins proflogistiche (IL-6, IL-8), angiotensinogen, plasminogen activator inhibitor-1, TNF- $\alpha$ and growth factors, species of vascular endothelial growth. Many of these factors are produced by stromo-vascular fraction of adipose tissue and macrophages infiltrating the adipose tissue. ${ }^{13}$

In addition to determining a proflogistic environment, the products of visceral adipocytes, unlike those of the subcutaneous tissue, have direct access to the liver, thus magnifying the negative consequences due to the excess of visceral fat. Many of these have a role in the relationship between central obesity, cardiovascular disease, dyslipidemia, type-two diabetes and systemic inflammation.

In diseases involving the subcutaneous adipose, it is reasonable to think that in tissue pain conditions, with no pathological evidence, the adipocytes can produce inflammation of factors, chemotactic factors and proteins of the complement cascade. ${ }^{14}$

\section{Diseases of the subcutaneous adipose tissue}

These annoying, sometimes disabling, diseases are rarely fatal, but mostly benign, therefore they have received little attention from mainstream medicine.

For the particular anatomical location of the adipose tissue and pathophysiological phenomena that involve local and systemic character, there is no specific medical branch of belonging, in the sense that the conditions which can affect the fat tissue may be attributable to the dermatologist, as well as the rheumatologist, as well as the internist and so on.

\section{Vasculitis}

The lesions of the microcirculatory units (MCUs) vessels are characterized by inflammatory processes affecting the wall of the arteries, veins and capillaries and other tissue components. ${ }^{13}$ The inflammatory process leads in most cases to immune pathogenesis, it can be characterized by the formation of immune complexes induced by heterologous antigens, such as viruses, bacteria, parasites autologous antigens such as Ig, DNA, neoplastic, of unknown nature, cell mediated hypersensitivity presenting necrotic infiltrative disorders, granulomatous manifestations and intermediate aspects.,13,14 In MCUs, as well as in large vessels, inflammation and degenerative processes can affect the arterial side as venous or both involving the system of the capillary network.

In general, the drugs used for the therapy of vasculitis are corticosteroids and antibiotics and this suggests the application of therapy with $\mathrm{O}_{2}-\mathrm{O}_{3}$ for the well-known anti-inflammatory properties as well immunomodulatory, anti-edema, static virus, bactericide, and for the ability to re-oxygenate the tissue and make the streets of the microcirculation more pervious to the passage of blood.

\section{Therapeutic actions ozone on microcirculation}

As before mentioned, the microcirculation, the surface of which has a development of about $6000 \mathrm{~m}^{2}$, consists of arterioles, capillaries and arterioles destination, which respectively have diameters of 20-50, 1015 and $5-9 \mu$. The flow in the capillary is controlled by sphincters pre capillaries where the blood, flowing, exchanges its nutritive factors, provides oxygen to the tissues and reabsorbs the products of cellular catabolism and carbon dioxide. The flow inside the capillary district is due to the pressure gradient between the arterial end, whose pressure is 32 $\mathrm{mmHg}$, and the venous end, which is of about $15 \mathrm{mmHg}$. The transit time of the erythrocytes is about 1-2 seconds in the capillaries, the length of which is about 0.10 to $0.15 \mathrm{~mm}$. The flow rate is very low if compared with that of the aorta and iliac where it is approximately 100 $\mathrm{cm}$ second. The red blood cell, which has a diameter of about 5-8 $\mu$, has normally a larger diameter of the capillary, to be able to cross it, deforming and adapting itself to the diameter of the vessel. According to the law of Poiselle the blood flow in a system of rigid pipes is directly proportional to the pressure difference between the extremes of the considered pipe and directly proportional to the fourth power of the radius. A small reduction of this determines the reduction of the flow, which results to be inversely proportional to the length of the vessel and the blood viscosity, that is, the resistance to flow and to the deformability of red blood cells. Blood viscosity is affected by hematocrit, the concentration of fibrinogen, by globulins suspended in plasma, and the deformability of red blood cells, as well as the aggregation of platelets and leukocytes, which are larger and more deformable than red blood cells.

$\mathrm{O}_{3}$ can be used in all forms of a disease in which there is a reduced supply of oxygen because it has an effect of reactivation of the microcirculation and multi organ protective action: i) peripheral arteriopathy of the lower limbs; ii) ischemic heart disease; iii) vascular renal impairment; iv) cerebrovascular diseases; v) maculopatia degenerative retinal.

$\mathrm{O}_{3}$ causes an increase in red cell deformability and filterability because it breaks the long chains of fatty acids controlled by lipid peroxidation, increases the production of 3.2 diphosphoglycerate with greater supply of oxygen to peripheral tissues by moving to the right of the hemoglobin dissociation curve, reduces platelet aggregation and plasma viscosity, reduces plasma fibrinogen, activates the energy metabolism in the mitochondria with increased production of ATP; it determines an increase of the blood flow, the release of nitric oxide at the level of pre-capillary sphincters by endothelial cells, it has an antinflammatory action resulting in a reduction in the production of prostaglandins and acting on Food on arachidonic acid, moreoves, it has an antioxidant action by activating protective antioxidant enzyme functions of endogenous cells. ${ }^{5-8,11}$

\section{Diseases of the subcutaneous adipose with mainly inflammatory and degenerative features}

These issues should deserve a much greater discussion, and in this paper I would like, altough in a non-exhaustive way, to take into account as main theme the panniculus injuries that sometimes characterize the framework of a large family of diseases involving the adipose tissue (Table 1).

\section{Erythema nodosum}

Erythema nodosum is a seasonal disease (spring, fall), common in young women, characterized by the appearance of skin nodules mostly in the legs (front part of the legs), and more rarely in the upper limbs and buttocks. ${ }^{4}$

The disease, with cast successors can last 4-6 weeks and is preceded by a general framework of symptoms characterized by fever, arthralgia, gastrointestinal disorders, tonsillitis, etc. In the resolution period, the general symptoms subside and skin lesions change their color to reddish or purplish red in blue, yellow-green and finally yellow (look bruised forms) reabsorbing without traces.

Erythema nodosum is an affection of the deep dermis and subcutaneous tissue on the basis of vasculitis with important allergic component ${ }^{4}$ which recognizes multiple and varied causes. 
Skin lesions appear as multiple knots, four or more each limb, slightly prominent on the skin plans of the diameter from 1 to $8 \mathrm{~cm}$, with sharp boundaries, painful on palpation, a hard consistency with red or purplish red appearance that is thrown to externalize successively. The cycle of each element is $10-20$ days without results. Histologically, an infiltrate consisting primarily of neutrophils in the dermal and subdermal connective limits and in subcutaneous septa can be noticed. ${ }^{14}$ Vessels in charge of the wall, are infiltrative phenomena and endothelial proliferation. Characteristics of Miescher nodules affect the deep dermis and the subcutaneous fibrous septa (Table 2). ${ }^{13}$

The symmetry of the lesions, the general symptoms, the characteristic color change of the knots and especially the spontaneous resolution without scars are characteristic of erythema nodosum. ${ }^{4}$

The differential diagnosis with other diseases such as nodular expres- sion hardened erythema of Bazin, fungal tires, tuberculosis and leutiche, or rheumatic fever, where, however, subcutaneous nodular lesions, which are very rare in adults, are small, hard, not sore and not inflamed. ${ }^{14}$

The causes have to be excluded for the first streptococcal infection (title ASLO streptozyme tests, throat swab) and sarcoidosis [RX chest, angiotensin converting enzyme dosage serum, eye examination (uveitis)].

In children before the age of five, most cases were secondary to tuberculosis, while after ten the most common cause and streptococcal infection was beta hemolytic group. Other causes have to be found in inflammatory bowel disease and upper airway.

\section{Classical therapy}

Causal therapy: eliminate antigen or drug; symptomatic treatment:

Table1. Diseases of connective and subcutaneous adipose tissue.

\begin{tabular}{ll}
\hline Panniculitis or Hypodermatitis & Christian-Weber panniculitis (rare) \\
& Hypodermitis stasis (common) \\
& Erythema nodosum (common) \\
& Bazin hardened erythema (rare) \\
& Solitary lipomas (common) \\
& Symmetrical lipomas in lipodystrophy cerebrospinal chest: \\
& - supraclavicular \\
& - trochanteric \\
Lipomatosis & - intra periarticular of knees, legs and ankles, (all common) \\
& Madelung disease (rare) \\
& Dercum syndrome (painful lipomatosis, rare) \\
& Universal diffuse lipomatosis (rare) \\
& Hoffman-Zurhelle syndrome (rare) \\
& Teutschlander syndrome or calcinosis lipogranulomatosis progressive justa-articular (elbows, rare), also it affects late teens \\
& Women cellulitis or PEFS alteration of subcutaneal fat (very common) \\
\hline
\end{tabular}

Table 2. Etiological factors of erythema nodosum.

\begin{tabular}{ll}
\hline Bacterial infections & Streptococcus pyogenes (very frequent) \\
& TBC (frequent) \\
& Sifilis (rare) \\
& Psittacosis (clamydie infections, not frequent) \\
& Diphtheria (rare) \\
& Leprosy (rare) \\
& Meningococcal Infection (rare) \\
& Yersinia enterolitica \\
& Cat-scratch disease (rare) \\
& Lymphogranuloma venereum (rare) \\
& Echo o Coxsackie \\
Virus infections & Filiarosis (rare) \\
Metazoans infestations & Ascaridosis (rare) \\
& Blastomycosis (rare) \\
Fungi infestations & Coccidioidomycosis (rare) \\
& Histoplasmosis (rare) \\
\hline Hypersensitivity reaction to drugs & Oral contraceptives \\
& Penicillin \\
& Sulfonamide \\
& Sarcoidosis (frequent) \\
Other diseases & Ulcerative colitis (frequent) \\
& Polyarteritis nodosa (rare) \\
& Systemic lupus erythematosus (rare) \\
& Bechcet disease (rare) \\
\end{tabular}


acetylsalicylic acid 2-2.5 g/day with gastroprotection misoprostol 400 $\mathrm{mg} /$ day; methylprednisolone $16 \mathrm{mg} /$ day per os in the morning to reduce slowly, to prevent relapse, of about $2.4 \mathrm{mg}$ for once during the first months and then $1 \mathrm{mg}$ per once every 20/30 days Finnic to the suspension within $3 / 6$ months; any elastic bandage for edema.

\section{Therapy with oxygen-ozone}

Therapy $\mathrm{O}_{2}-\mathrm{O}_{3}$ must take account of vasculitic component, inflammatory and edema of the lesions. The clinical picture suggests the local therapy as the systemic one, while according to the causes above mentioned, as shown in the Tables 1 and 2, it can be considered both the antibacterial, antiviral and immunomodulating action. The abilities to improve the hypoperfusion and hypometabolism, the tissue ischemia, acute-chronic course of injuries and relapses, suggest protocols characterized by choices that consider the immunological component but also anti-inflammatory and anti-edema ones. The clinical picture suggests therapeutic choices geared toward systemic treatments with granulomatous amebic encephalitis interspersed by prostate artery embolization and the local mes otherapy but also with rectal insufflation (Table 3).

\section{Hypodermitis stasis}

The phlebological Hypodermatitis recognizes increased capillary permeability, edema and lymphatic stasis as primary causes. ${ }^{4}$ Some forms also recognize pathological events from the scene of the observable evidence that most of the time are clinically asymptomatic and not yet manifested, as in cardiocirculatory, liver and kidney diseases. While others have an acute onset that can be triggered by physical and/or mental trauma, by exposure to heat or prolonged immobility in a standing or sitting position, where the lymphatic and venous return is obstructed by the failure of the muscle or foot pump, or from the wrong posture. Among the most common causes we can include a diet too rich in salts, premenstrual syndrome, pregnancy, or taking medicines, especially for high blood pressure, nonsteroidal anti-inflammatory drugs (NSAIDS), steroids, estrogens.

In the doldrums the trans capillary passage of proteins (albumin, fibrinogen, Ig) is increased. Fibrinogen, which accumulates in large quantities in the tissue, seems to form true pericapillary sleeves fibrin, causing an inflammation in macrophage and lymphocyte that is the basis of pain and inflammation in hypodermatitis.

A certain amount of white blood cells and T-lymphocytes is retained in the tissue and, for their difficulty in passing through the wall of the capillary, they remain on site attached to the vessel wall by releasing the granules containing proteolytic enzymes. In particular, the activated macrophages that produce IL-1, which stimulates endothelial cells to produce plasminogen inhibitor activator, may explain the reduced fibrinolytic activity, which occurs in patients with chronic venous insufficiency. ${ }^{4,13}$ The rheological alteration and dependant modifications of endothelial cells lead to increased platelet adhesion and activation with production and release of TNF- $\alpha$, which is the key cytokine for tissue repair, whose protracted production determines the development of the fibrous septa.

The reduced fibrinolytic activity facilitates the precipitation and the persistence of fibrinogen in the tissue increasing the activity.

After the acute phase, in chronic edema, hypodermitis evolves towards a phase of retractable fibrosis sclerodermiform affecting the deep tissues, and the adipose tissue disappears replaced by a dense tissue. Initially the hypodermitis looks like an indurate plate, round or oval, subcutaneous, supra ankle, adhering to both the surface and deep plans, painful on palpation. It presents clear signs of inflammation, redness and heat of the skin.

The pain can be spontaneous, even at night, exacerbated by walking and standing position.

Sometimes the nodules can be spread, which gives the need for a differential diagnosis of erythema nodosum. At this stage there is a lack of phlebitis, infectious complications (erysipelas), even if there are venous and lymph stasis. ${ }^{4,14}$ The first symptom is pain on palpation without appreciable hardening of the part. The concerned segment presents elastic edema whose result will be hardening, petechial hemorrhages, ocher dermatitis, white patches of Milian atrophy and finally necrotic reactions and the formation of an ulcer maybe as a result of minor injuries.

In the final stage we see the sclerosing dermatitis, atrophy with pigmentary and sclero-hypertrofic leg characterized by a concrete block dermatitis that holds the ankle in the area where there are calcifications and subcutaneous ossifications.

\section{Classical therapy}

Physical therapy: i) compression bandages - elastic bandage for $3 / 6$ months; ii) daily manual lymphatic drainage followed by a strong compression elastic stockings or compression bandage.

Pharmacological therapy: i) venotropic drugs: diosmin $500 \mathrm{mg} \times 2 /$ day

Table 3. Prospectus therapy with oxygen-ozone in the erythema nodosum.

\begin{tabular}{|c|c|c|c|c|c|}
\hline Concentration $\mathrm{O}_{2}-\mathrm{O}_{3}$ & Tecnique & Syringe & Needle & Weekly sessions & Total sessions \\
\hline $3-5 \mu g$ & $\begin{array}{l}\text { Local perivenous infiltrations } \\
\qquad 50 \mathrm{cc}\end{array}$ & $20 \mathrm{cc}$ & $\begin{array}{c}27 \mathrm{~g} \\
4 \mathrm{~mm}\end{array}$ & $2 / 3$ & $12 / 24 / 36$ \\
\hline $20-40 \mu g$ & $\begin{array}{l}\text { GAE/systemic ossigenation } \\
150 \text { cc } \mathrm{O}_{2}-\mathrm{O}_{3}+200 \text { blood }\end{array}$ & $50 \mathrm{cc}$ & l & 2 & $\begin{array}{c}12 / 24 / 36 \\
+ \text { one weekly till the healing of lesions }\end{array}$ \\
\hline $50-5 \mu g$ & $\begin{array}{l}\text { Topical treatment } \\
\text { with bag in case of ulcers }\end{array}$ & l & 1 & $2 / 3$ & $12 / 24$ \\
\hline $10-20 \mu g$ & Rectal insufflation & $50 \mathrm{cc}$ & I & $1 / 2$ & $12 / 24 / 36$ \\
\hline \multicolumn{6}{|c|}{ Om3 long-life water 2-3 glasses/day } \\
\hline \multicolumn{6}{|c|}{ Applicate ozoven } \\
\hline \multicolumn{6}{|c|}{ Balanced diet rich in flavonoids } \\
\hline \multicolumn{6}{|c|}{ Moderate physical activity - walking on a treadmill with orthopedic belt (thickness 3-4.2 mm) } \\
\hline
\end{tabular}

GAE, granulomatous amebic encephalitis. 
or oxerutins $1000 \mathrm{mg} \times 2-3 /$ day, or dihydroergotamine $3 \mathrm{mg} \times 3 /$ day; ii) NSAIDS: nimesulide $100 \mathrm{mg} \times 2 /$ day; or ibuprofen $600 \mathrm{mg} \times 2-3 /$ day. Periods of 1/2 weeks associated with misoprostol $400 \mathrm{mcg} / \mathrm{day}$; iii) corticosteroids: betamethasone sodium phosphate $2 \mathrm{mg} /$ day for the first 3 days and then $0.5 \mathrm{mg} /$ day for 4 days.

Surgical therapy: i) permanent discontinuation of the intervention saphenofemoral, ligation of perforating veins and sclerosing injections; ii) saphenectomy are in the elderly.

Treatment of infectious complications: antibiotics in pushed lymphangitis that erysipeloid: amoxicillin trihydrate $1 \mathrm{~g} \times 2 /$ day for at least 10 days.

Therapy of varicose ulcers: i) prolonged rest with leg raised to facilitate venous outflow; ii) antibiotics; iii) local dressings with products such as cadexomer iodine and/or dextran with epichlorohydrin or dimeticone (Table 4).

\section{Localized lipodystrophy - panniculopathy edematous fibro sclerotic}

Today the misnomer of cellulite includes some blemishes whose nature and causes require, sometimes, therapeutic intervention of the doctor. He has the duty to care for the body and also the tissues that compose it, respecting its integrity and functions without putting in place practices that can cause pain, damage or cell death. The $\mathrm{O}_{2}-\mathrm{O}_{3}$ therapy centers refine these objectives, acting as a medical practice whose only purpose is to heal and to restore cellular homeostasis and tissue function. ${ }^{16}$

For PEFS we mean an alteration of the subcutaneous fat to a regressive character of unknown etiology. As previously noted in women, the tips of the lobules, which have a spherical shape, press against the reticular dermis and protrude in the form of fat buds characterizing, with a hilly profile, the dermo-hypodermic border. The branches of connective tissue that surround and hold the lobules are disposed perpendicularly to the skin level, which, in case of adiposity, conveys the acquired volume to the overlying buds, causing uneven skin surface depressions (orange peel skin) ${ }^{15}$

This benign disease occurs almost exclusively in women and occurs in all ages, according to the different forms that characterize it. It is localized predominantly in the anterior-lateral regions of the thighs and calves but also in the abdominal region and the rear of the arms.

We need to distinguish it from the fatty deposits like pertrochanteric bearings, due to a mechanical reaction to the gain of body weight, for prolonged sitting position, where we can observe a strong accumulation of fat, with the subversion of the architectural structure of the tissue, the suffering of the microcirculation, the presence of telangiectasic tufts, sclerosis of the connective stromal component, sometimes modest inflammation and in severe cases cold skin for reduced perfusion and metabolic activity. In cellulitis we can distinguish an inflammatory form, an edematous, fibrous and sclerotic. These correspond to the different developmental stages of suffering and histological tissue. The inflammatory edematous form seems to be typical of young age.

Among the causes that can trigger the phenomena, which lead to the panniculosis, are the stagnation of the microcirculation, which causes an increase of albumin protein, fibrinogen and immunoglobulins' passage. ${ }^{15}$ The fibrinogen can wrap the capillaries, as a sleeve of fibrin, partially occluding the pinocytosis channels. This causes the marginalization of the white blood cells which, for the greater difficulties in which they deform, pass the wall with difficulty increasing the vascular resistance. The polymorphic leukocytes attached to the endothelium can be activated releasing their granules, which contain proteolytic enzymes. The increased presence of tissue macrophages and T-lymphocytes stimulates IL-1 production that induces endothelial cells to synthesize plasminogen activator inhibitor, which may explain the reduced fibrinolytic activity and which is found in the venous failure.

Although there isn't an extensive experimental research, it has been estimated that the doldrums, the rheological alteration and alteration of endothelial cells, involve an increase in the phenomenon of adhesion, with the production and release of TGF- $\beta$, which produced in a protracted time, can determine diffuse fibrosis. ${ }^{15}$ The reduced fibrinolytic activity facilitates the precipitation and the persistence of fibrinogen in the interstitial tissue, increasing the activity of fibroblasts. After the acute phase, in chronic edema, hypodermitis evolves toward the retractable fibrosis. The question that arises is: what is the real state of disease or tissue affliction, relatively to the aforementioned processes and intensity of symptoms?

To date, the only honest response can be made merely from observation and experience, as there are no experimental studies and extensive research on the topic.

\section{Predisposing factors}

Among the predisposing factors we include muscular hypotonia, postural defects, incorrect plantar support, excessive localized fat, disordered lifestyles, unbalanced nutrition with low-protein or hypovitaminic and low in fiber diet, prolonged standing, use of contraceptives where estrogens cause an increase in water retention and the patency of capillaries, a sedentary lifestyle, obesity.

The muscular hypotonia intervenes negatively on the pump functions of the arterial and venous vessels accentuating the doldrums in the lymphatic system, which does not fully perform the functions of drainage,

Table 4. Statement of therapy with oxygen-ozone in hypodermitis stasis.

\begin{tabular}{|c|c|c|c|c|c|}
\hline Concentration $\mathrm{O}_{2}-\mathrm{O}_{3}$ & Tecnique & Syringe & Needle & Weekly sessions & Total sessions \\
\hline $3-5 \mu g$ & $\begin{array}{l}\text { Local perivenous infiltrations } \\
\qquad 50 \mathrm{cc}\end{array}$ & $20 \mathrm{cc}$ & $\begin{array}{c}27 \mathrm{~g} \\
4 \mathrm{~mm}\end{array}$ & $2 / 3$ & $12 / 24 / 36$ \\
\hline $20-40 \mu g$ & $\begin{array}{l}\text { GAE/systemic ossigenation } \\
150 \text { cc } \mathrm{O}_{2}-\mathrm{O}_{3}+200 \text { blood }\end{array}$ & $50 \mathrm{cc}$ & I & 2 & $\begin{array}{l}12 / 24 / 36 \\
+ \text { one weekly till the healing of lesions }\end{array}$ \\
\hline 50-5 $\mu \mathrm{g}$ & $\begin{array}{l}\text { Topical treatment } \\
\text { with bag in case of ulcers }\end{array}$ & / & / & $2 / 3$ & $12 / 24$ \\
\hline $10-20 \mu g$ & Rectal insufflation & $50 \mathrm{cc}$ & I & $1 / 2$ & $12 / 24 / 36$ \\
\hline \multicolumn{6}{|c|}{$\mathrm{Om}_{3}$ long-life water 2-3 glasses/day } \\
\hline \multicolumn{6}{|c|}{ Applicate ozoven } \\
\hline \multicolumn{6}{|c|}{ Balanced diet rich in flavonoids } \\
\hline \multicolumn{6}{|c|}{ Moderate physical activity - walking on a treadmill with orthopedic belt (thickness 3-4.2 mm) } \\
\hline
\end{tabular}

GAE, granulomatous amebic encephalitis. 
transportation, exchange of nutrients and waste, transport of leukocytes in lymphatic capillaries, elimination of pathogenic microorganisms, filtration, purification of the lymph and so on.

Postural abnormalities may determine: i) hyperstanding on a limb, causing muscle contraction and sometimes spasm with changes on the dynamics of the venous lymphatic circulation, accentuating the phenomenon of stasis; ii) incorrect plantar support with cavus (high-heeled shoes) or flat-footedness of the foot that determines in walking the wrong pressing of the base of the foot pump with dysfunction of the lymphatic venous circulation return.

Another predisposing factor is the sedentary life, the effects of the diet on the energy balance of the body, for important metabolic and dynamic meanings on the blood and lymphatic circulation. ${ }^{14,15}$

The movement in the lower limbs in the veins and lymphatic vessels is against gravity where the centripetal flow opens the semilunar venous valves and the centrifugal one closes them.

Venous blood is driven from the periphery to the center by the forces acting from behind, that is, by the activity of vermicular systolic-diastolic movements transmitted from neighboring arteries to the vein wall, by squeezing the Lejard's venous sole, the muscle pump, by those forces acting from the front represented by the diaphragmatic excursion, which by compressing and decompressing the intestinal package implements reflux and suction mechanisms creating negative pressure gradient.

\section{Classification of panniculopathy edematous fibro sclerotic}

Commonly it is used to describe the various stages in the progression of the formation of cellulite: i) congestive phase: venous and lymphatic stasis with poor tissue oxygenation, impaired drainage of interstitial fluids, increase in the volume of adipocytes, and interstitial inflammation serous, cold skin and edematous; ii) exudative phase: the epidermis thins becoming brittle and dehydrated. The suffering of the microcirculation increases with the infiltration of polymorph elements nucleated with fibrinous inflammation in the initial phase, micro nodules formation; the skin is slightly reddened in patches, ${ }^{4}$ iii) fibrous phase: persistent inflammation and edema, leakage of fibrinogen generates the production of fibrin into the gap that represents a powerful stimulus to the repair by stimulating fibroblasts to lay down collagen fibers, which determine the beginning of the structural disruption of the tissue with formation of small adipocyte clusters (nodules), ${ }^{4,15}$ pain caused by palpation; iv) phase of fibrous scar: you can appreciate subversion of the lobular structure of the adipose tissue, ${ }^{15}$ formation of painful nodules on palpation, sometimes spontaneous pain, even at night, with ankle edema. The evolution of the disease also leads to symmetrical changes in the so-called orange peel profile, and skin hypothermia, poor dermographism, stretch marks and spider veins, feeling of cold, heaviness, local paresthesia can be detected and also observed.

\section{The alterations of the vessels and blood flow in panniculopathy edematous fibro sclerotic}

The microcirculatory system basically consists of a series of branched canaliculi, anastomosed with each other, covered with endothelium, the flow is regulated to arteriolar level at each moment, only a few capillaries are open, the others remain closed; then, to vary the flow to the tissue, the true capillaries do not dilate, but open to a greater number simultaneously.

After a damaging action with inflammation in the microcirculation, it manifests arteriolar dilation, sometimes preceded by a very short interval of vasoconstriction, all the precapillary sphincters open simultaneously and dilate the postcapillary venules filling quickly with blood all over the local microcirculation expanding and filling with blood.

Vascular changes, hyperemia and increased blood flow are followed by the slowing of blood flow, increased intra-vascular pressure, decreased drainage, increased hydrostatic pressure in capillaries and venules. This causes a modification of the provision of corpuscular elements in the vessels where the reduced flow speed pushes the red blood cells to assume a central position, while the white blood cells, especially neutrophils, tend to set peripherally to the lumen, helping to obstruct the drainage of blood from the same affected districts causing stasis. The altered vascular permeability, the increasing of the hydrostatic pressure and the phenomena that involve the entire cell population determine exudation with liquid containing plasma proteins and leukocytes leaking.

\section{Mechanisms of action of oxygen-ozone in panniculopathy edematous fibro sclerotic}

The $\mathrm{O}_{3}$ working mechanisms in inflamed tissue stasis of microcirculation, edema and vasculitis-related phenomena are: i) the unsaturated fatty acid that is related to the double carbon bond transformed into water-soluble compounds; ii) $\mathrm{O}_{3}$ that develops an interaction with the double bonds of unsaturated fatty acids of the phospholipids in the erythrocyte membrane layer by increasing the deformability. ${ }^{5,7}$ In this way it reduces blood viscosity and increases the production of 2.3-diphos-

Table 5. Therapy with oxygen-ozone in lipodystrophies.

\begin{tabular}{|c|c|c|c|c|c|}
\hline Concentration $\mathbf{O}_{2}-\mathbf{O}_{3}$ & Tecnique & Syringe & Needle & Weekly sessions & Total sessions \\
\hline $\begin{array}{l}\text { 1-5 ug max } \\
\text { Hard: } 1.5-2.5 \\
\text { Soft: } 2-3 \\
\text { Edematous: } 4-5\end{array}$ & $\begin{array}{l}\text { Subcutaneous infiltration } \\
5-7 \text { cc point } \\
\text { Max } 200 \text { per session }\end{array}$ & $50 \mathrm{cc}$ & $\begin{array}{c}27 \mathrm{~g} \\
4 \mathrm{~mm} \text { or } \\
26 \mathrm{~g} 12 \mathrm{~mm}\end{array}$ & $2 / 3$ & $12 / 24$ \\
\hline $10-20 \mu g$ & Rectal insufflation & $50 \mathrm{cc}$ & l & $1 / 2$ & 12 \\
\hline Ozoil body & $\begin{array}{l}\text { Lymphatic drainage massage } \\
\text { release of lymph node stations }\end{array}$ & / & / & $1 / 2$ & $12 / 24$ \\
\hline \multicolumn{6}{|c|}{ Om3 long-life water 2-3 glasses/day } \\
\hline \multicolumn{6}{|c|}{ Physical activity immediately after treatment by calculating the heart rate for lipolytic activity } \\
\hline \multicolumn{6}{|c|}{ Balanced diet low in sodium } \\
\hline \multicolumn{6}{|c|}{ Moderate physical activity - walking on a treadmill with orthopedic belt (thickness 3-4.2 mm) } \\
\hline & & Sequen & pressure air & & \\
\hline
\end{tabular}


phoglycerate,,${ }^{5,7}$ responsible for the transfer of $\mathrm{O}_{2}$ from hemoglobin to tissues, with better perfusion and transport along the vascular beds; iii) $\mathrm{O}_{3}$ that exerts anti-inflammatory action by reducing the production of pro-inflammatory cytokines, immunoglobulins and inflammatory mediators. ${ }^{5,7-9,11,17,18}$

\section{Oxygen-ozone therapy in lipodystrophy}

For therapy we have to consider the evolution, the vascular impairment, the congestive pain, the edema, the inflammation, and degenerative and sclerotic widespread phenomena. We also analyze the tissue architecture subversion, all factors that characterize the framework and symptoms, as well as the psychological conditions of the patient, the weight, muscle tone and nutritional status (Table 5).

\section{Conclusions}

With this short dissertation, I have tried to show, through a deductive reasoning starting from some scientific observations as well as learning from clinical indications of talented colleagues, how you can get, in medical practice, a positive experience, but that is certainly not exhaustive of the subject matter.

\section{References}

1. Bittolo Bon G. Il tessuto adiposo come organo multifunzionale. G Ital Cardiol 2008;923S-8S.

2. Tedesco L, Carrubba M0, Nisoli E. Tessuto adiposo come organo endocrino. G It Diabetol Metab 2008;28:90-100.

3. Lanza G. Anatomia patologica sistematica. $2^{\text {nd }}$ ed. Padova: Piccin Nuova Libraria; 1985.

4. Martinez Braga G, Di Martino Ortiz B. Septal panniculitis: clinicopathological review of literature and case presentation. Our Dermatol Online 2011;5:74-82.
5. Valdenassi L, Franzini M, Richelmi P, Berte F. Ossigeno-ozono terapia. Proceedings of the $1^{\text {st }}$ Congress of the SIMFER Section of Ambulatorial Rehabilitation. 2003 Nov 22, Pavia, Italy.

6. Valdenassi L, Franzini M, Simonetti V, Ricevuti G. Oxygen-ozone therapy: paradoxical stimulation of ozone. Ozone Therapy 2016;1:5837.

7. Sagai M, Bocci V. Mechanisms of action involved in ozone therapy: is healing induced via a mild oxidative stress? Med Gas Res 2011;1:29.

8. Bocci V. Ossigeno ozonoterapia. Comprensione dei meccanismi d'azione e possibilita terapeutiche. Rozzano, MI: Casa Editrice Ambrosiana; 2000.

9. Bocci V, Zanardi I, Travagli V. Oxygen/ozone as a medical gas mixture. A critical evaluation of the various methods clarifies positive and negative aspects. Med Gas Res 2011;1:6.

10. Al-Hajjar N. The effect of ozonized saline solutions processed under intense electric fields in the treatment of infected necrotiz ing acute pancreatitis: an experimental mode. Int $\mathrm{J}$ Med Medical Sci 2014;6:176-82.

11. Bocci V, Zanardi I, Travagli V. Ozone acting on human blood yields a hormetic dose-response relationship. J Trans Med 2011;9:66.

12. Simonetti V, Liboni W, Molinari F. Why ozone therapy in multiple sclerosis? Rev Esp Ozonoterapia 2014;4:51-68.

13. Yi SW, Kim EH, Kang HY, et al. Erythema nodosum: clinicopathologic correlations and their use in differential diagnosis. Yonsei Med J 2007;48:601-8.

14. Chan MP. Neutrophilic panniculitis: algorithmic approach to a heterogeneous group of disorders. Arch Pathol Lab Med 2014;138:1337-43.

15. Terranova F, Berardesca E, Maibacha H. Cellulite: nature and aetiopathogenesis. Int J Cosmet Sci 2006;28:157-67.

16. Tylicki L, Nieweglowski T, Biedunkiewicz B, et al. The influence of ozonated autohemotherapy on oxidative stress in hemodialyzed patients with atherosclerotic ischemia of lower limbs. Int $\mathrm{J}$ Artif Organs 2003;26:297-303.

17. Borrelli E, De Monte A, Bocci V. Oxygen ozone therapy in the integrated treatment of chronic ulcer: a case series report. Int J Recent Sci Res 2015;6:4132-6.

18. Thakkar V, Thakkar H. Ozone (03): an excellent adjunctive tool in medical and surgical management of patient. Int J Res Med Sci 2014;2:1257-61. 